Journal of Applied AnALysis

Vol. 8, No. 2 (2002), pp. 201-219

\title{
ON DENSITY TOPOLOGIES WITH RESPECT TO INVARIANT $\sigma$-IDEALS
}

\author{
J. HEJDUK \\ Received June 13, 2001 and, in revised form, December 17, 2001
}

\begin{abstract}
The density topologies with respect to measure and category are motivation to consider the density topologies with respect to invariant $\sigma$-ideals on $\mathbb{R}$. The properties of such topologies, including the separation axioms, are studied.
\end{abstract}

\section{Notation}

By $\mathbb{R}$ we shall denote the set of all reals numbers and by $\mathbb{N}$ the set of positive integers. Let $l$ stand for Lebesgue measure. The capitals $\mathcal{L}$ and $\mathbb{L}$ denote the $\sigma$-algebra of all Lebesgue measurable sets in $\mathbb{R}$ and the $\sigma$-ideal of all Lebesgue null sets. The natural topology on $\mathbb{R}$ is denoted by $\mathcal{T}_{0}$. If $\mathcal{T}$ is a topology on $\mathbb{R}$, then we fix the notation:

$\mathcal{B}(\mathcal{T})$ - the $\sigma$-algebra of all Borel sets with respect to $\mathcal{T}$,

$\mathcal{B} a(\mathcal{T})$ - the $\sigma$-algebra of all sets having the Baire property with respect to $\mathcal{T}$,

$\mathcal{K}(\mathcal{T})$ - the $\sigma$-ideal of all meager sets with respect to $\mathcal{T}$.

2000 Mathematics Subject Classification. 28A05, 54A10.

Key words and phrases. Density point, density topology, the separation axioms, invariant ideals and algebras.

ISSN 1425-6908 (C) Heldermann Verlag. 
For any set $X \subset \mathbb{R}, \operatorname{Int}_{\mathcal{T}} X$ is the interior of $X$ with respect to $\mathcal{T}$, and $\bar{X}^{\mathcal{T}}$ is the closure of $X$ with respect to $\mathcal{T}$. If $\mathcal{T}=\mathcal{T}_{0}$, then we use shortly the following symbols: $\mathcal{B}, \mathcal{B} a, \mathbb{K}, \operatorname{Int} X, \bar{X}$. The symmetric difference of sets $X$ and $Y$ we shall denote by $X \triangle Y$, and $S \triangle \mathcal{J}$ denotes the smallest $\sigma$-algebra containing $S$ and $\mathcal{J}$. For any sets $X$ and $Y$ belonging to $S$, the fact that $X \triangle Y \in \mathcal{J}$ will be denoted by $X \sim Y$. For each set $X \subset \mathbb{R}$ and $a, t \in \mathbb{R}$, we denote

$$
\begin{aligned}
t X & =\{y \in \mathbb{R}: y=t x, x \in X\}, \\
X+a & =\{y \in \mathbb{R}: y=x+a, x \in X\} .
\end{aligned}
$$

By $\mathcal{J}_{0}$ we shall denote the ideal consisting of the empty set, and by $\mathcal{J}_{\omega}$ the $\sigma$-ideal of the countable sets. Only proper $\sigma$-ideals are considered. The cardinality of the continuum is denoted by $\mathfrak{c}$.

\section{The concept of the density topology}

Let $X \in \mathcal{L}$. We say that 0 is a Lebesgue density point of $X$ if $\lim _{h \rightarrow 0^{+}} l(X \cap[-h, h]) /(2 h)=1$. It is not difficult to check that the last assertion is equivalent to the statement saying that $\lim _{n \rightarrow \infty} l(n X \cap$ $[-1,1])=2$. This is equivalent to the fact that the sequence of characteristic function $\left\{f_{n}\right\}_{n \in \mathbb{N}}=\left\{\chi_{n X \cap[-1,1]}: n \in \mathbb{N}\right\}$ tends in measure to $\chi_{[-1,1]}$ (see $[15]$ ). Using the Riesz theorem, we obtain that the sequence $\left\{f_{n}\right\}_{n \in \mathbb{N}}$ converges with respect to the $\sigma$-ideal of the Lebesgue null sets. It means that every subsequence of the sequence $\left\{f_{n}\right\}_{n \in \mathbb{N}}$ contains subsequence convergent to $\chi_{[-1,1]}$ almost everywhere.

The concept of convergence with respect to a $\sigma$-ideal (see [14]) enables one to introduce a density point with respect to the Baire category (see [13], $[15],[16])$. We extend this concept to consider the density topologies with respect to invariant $\sigma$-ideals.

Definition 1.1. We shall say that a family $\mathcal{A}$ of subsets of $\mathbb{R}$ is invariant if for each $X \in \mathcal{A}$ and all $n \in \mathbb{N}, a \in \mathbb{R}$, we have that $n X \in \mathcal{A}$ and $X+a \in \mathcal{A}$.

Definition 1.2. We shall say that a pair $(\mathcal{S}, \mathcal{J})$, where $\mathcal{S}$ is a $\sigma$-algebra of subsets of $\mathbb{R}$ and $\mathcal{J}$ is a $\sigma$-ideal of subsets of $\mathbb{R}$, is invariant if $\mathcal{J} \subset \mathcal{S}$, and both the $\sigma$-algebra $\mathcal{S}$ and the $\sigma$-ideal $\mathcal{J}$ are invariant.

We consider only invariant pairs $(\mathcal{S}, \mathcal{J})$ such that $\mathcal{B} \subset \mathcal{S}$.

Remark 1.3. If $\mathcal{J}$ is an invariant $\sigma$-ideal, then the pair $(\mathcal{B} \Delta \mathcal{J}, \mathcal{J})$ is invariant.

From now, let $(\mathcal{S}, \mathcal{J})$ be an invariant pair. 
Definition 1.4. We shall say that 0 is a $\mathcal{J}$-density point of an $\mathcal{S}$-measurable set $X$ if and only if the sequence of characteristic functions $\left\{\chi_{n X \cap[-1,1]}\right.$ : $n \in \mathbb{N}\}$ is convergent with respect to the $\sigma$-ideal $\mathcal{J}$ to the characteristic function $\chi_{[-1,1]}$ (it means that every subsequence of the sequence $\chi_{[-1,1]}$ contains a subsequence convergent to $\chi_{[-1,1]}$ everywhere except for a set belonging to $\mathcal{J}$.

A point $x_{0} \in \mathbb{R}$ is a $\mathcal{J}$-density point of a set $X \in \mathcal{S}$ if and only if 0 is a $\mathcal{J}$-density point of the set $X-x_{0}$.

For each $X \in \mathcal{S}$, we define

$$
\Phi_{\mathcal{J}}(X)=\{x \in \mathbb{R}: x \text { is a } \mathcal{J} \text {-density point of } X\} .
$$

The following property is an easy and useful characterization of the fact that 0 is a $\mathcal{J}$-density point of the set $X$.

Lemma 1.5 (cf. [3], [15]). The number 0 is a $\mathcal{J}$-density point of the set $X \in \mathcal{S}$ if and only if, for each increasing sequence $\left\{n_{k}\right\}_{k \in \mathbb{N}}$ of positive integers, there exists a subsequence $\left\{n_{k_{j}}\right\}_{j \in \mathbb{N}}$ such that

$$
\limsup _{j \rightarrow \infty}\left([-1,1] \backslash n_{k_{j}} X\right) \in \mathcal{J} \text {. }
$$

It is clear that the last condition has the form

$$
\bigcap_{i=1}^{\infty} \bigcup_{j=i}^{\infty}\left([-1,1] \backslash n_{k_{j}} X\right) \in \mathcal{J} .
$$

Directly from the definition of a $\mathcal{J}$-density point we have

Proposition 1.6. For every $\mathcal{S}$-measurable set $X$, every positive integer $n$ and every real number $a$, if $x \in \Phi_{\mathcal{J}}(X)$, then $n x \in \Phi_{\mathcal{J}}(n X)$ and $(x+a) \in$ $\Phi_{\mathcal{J}}(X+a)$.

Proposition 1.7. For any $\mathcal{S}$-measurable sets $X$ and $Y$, if $X \subset Y$, then $\Phi_{\mathcal{J}}(X) \subset \Phi_{\mathcal{J}}(Y)$.

As a consequence of the definition of a $\mathcal{J}$-density point we have for each $\sigma$-ideal $\mathcal{J} \subset S$ the following three propositions:

Proposition 1.8. For any $\mathcal{S}$-measurable sets $X$ and $Y$, the following conditions hold:

I. if $X \sim Y$, then $\Phi_{\mathcal{J}}(X)=\Phi_{\mathcal{J}}(Y)$,

II. $\Phi_{\mathcal{J}}(X \cap Y)=\Phi_{\mathcal{J}}(X) \cap \Phi_{\mathcal{J}}(Y)$,

III. $\Phi_{\mathcal{J}}(\emptyset)=\emptyset, \Phi_{\mathcal{J}}(\mathbb{R})=\mathbb{R}$. 
We define the family $\mathcal{T}_{\mathcal{J}}$ of $\mathcal{S}$-measurable sets by

$$
\mathcal{T}_{\mathcal{J}}=\left\{X \in \mathcal{S}: X \subset \Phi_{\mathcal{J}}(X)\right\} .
$$

Propositions 1.6 and 1.8 imply

Proposition 1.9. The family $\mathcal{T}_{\mathcal{J}}$ has the following properties:

1. $\emptyset, \mathbb{R} \in \mathcal{T}_{\mathcal{J}}$,

2. $\mathcal{T}_{\mathcal{J}}$ is closed under finite intersections,

3. if $X \in \mathcal{J}$, then $\mathbb{R} \backslash X \in \mathcal{T}_{\mathcal{J}}$,

4. $\mathcal{T}_{\mathcal{J}}$ is invariant with respect to each operation of the form $n x+a$ where $n \in \mathbb{N}$ and $a \in \mathbb{R}$.

We are also pointing out the following

Proposition 1.10. $\mathcal{T}_{0} \subset \mathcal{T}_{\mathcal{J}}$.

Proof. Let $V_{0} \in \mathcal{T}_{0}$. Of course, $V \in \mathcal{S}$. If $V=\emptyset$, then, by condition III of Proposition 1.8, we have $V \in \mathcal{T}_{\mathcal{J}}$. Let $x_{0} \in V$. Then $0 \in V-x_{0}$. Since $V-x_{0}$ is open, there exists $\varepsilon>0$ such that $(-\varepsilon, \varepsilon) \subset V-x_{0}$. It is obvious that, for every increasing sequence $\left\{n_{i}\right\}_{i \in \mathbb{N}}$ of positive integers, $\bigcap_{j=1}^{\infty} \bigcup_{i=j}^{\infty}\left([-1,1] \backslash n_{i}\left(V-x_{0}\right)\right)=\emptyset$. This means that $x_{0}$ is a $\mathcal{J}$-density point of $V$. Since $x_{0}$ is an arbitrary point, we conclude that $V \in \mathcal{T}_{\mathcal{J}}$.

Although the family $\mathcal{T}_{\mathcal{J}}$ containing $\emptyset$ and $\mathbb{R}$ is closed under finite intersections, it need not be a topology on the real line.

Example 1.11. Let us consider the pair $\left(\mathcal{B}, \mathcal{J}_{\omega}\right)$. Obviously $\left(\mathcal{B}, \mathcal{J}_{\omega}\right)$ is an invariant pair. However, the family $\mathcal{T}_{\mathcal{J}_{\omega}}=\left\{X \in \mathcal{B}: X \subset \Phi_{\mathcal{J}_{\omega}}(X)\right\}$ is not a topology.

To prove this, we use the example given in Lemma 2.18 from [3]. Namely, there exists a perfect set $C \subset \mathbb{R}$ such that each number $x \in C$ is a $\mathcal{J}_{\omega}$-density point of the set $\mathbb{R} \backslash C$. Simultaneously, by Proposition 1.10, we have that $\mathbb{R} \backslash C \subset \Phi_{\mathcal{J}_{\omega}}(\mathbb{R} \backslash C)$. Hence $\Phi_{\mathcal{J}_{\omega}}(\mathbb{R} \backslash C)=\mathbb{R}$. Let $P$ be a non-Borel subset of $C$. If $x \in P$, then $\{x\} \cup(\mathbb{R} \backslash C) \in \mathcal{T}_{\mathcal{J}_{\omega}}$ because $\{x\} \cup(\mathbb{R} \backslash C) \in \mathcal{B}$ and $\{x\} \cup(\mathbb{R} \backslash C) \subset \Phi_{\mathcal{J}_{\omega}}(\{x\} \cup(\mathbb{R} \backslash C))$. But $\bigcup_{x \in P}(\{x\} \cup(\mathbb{R} \backslash C))=P \cup(\mathbb{R} \backslash C) \notin \mathcal{B}$.

Motivated by this example, we introduce the following

Definition 1.12. If the family

$$
\mathcal{T}_{\mathcal{J}}=\left\{X \in \mathcal{S}: X \subset \Phi_{\mathcal{J}}(X)\right\}
$$

forms a topology, then $\mathcal{T}_{\mathcal{J}}$ is called the $\mathcal{J}$-density topology associated with the pair $(\mathcal{S}, \mathcal{J})$ or the $\mathcal{J}$-density topology generated by the pair $(\mathcal{S}, \mathcal{J})$. 
Example 1.13. If $\mathcal{J}$ is an invariant $\sigma$-ideal, then the pair $\left(2^{\mathbb{R}}, \mathcal{J}\right)$ is invariant and, by Propositions 1.7 and 1.9 , we conclude that the family $\mathcal{T}_{\mathcal{J}}$ is a $\mathcal{J}$-density topology associated with the pair $\left(2^{\mathbb{R}}, \mathcal{J}\right)$.

The whole difficulty to prove that an invariant pair $(\mathcal{S}, \mathcal{J})$ generates a $\mathcal{J}$-density topology lies in the verification whether the family $\mathcal{T}_{\mathcal{J}}$ is closed under an arbitrary union. In Example 1.13 we could avoid this difficulty because of the fact that $\mathcal{S}=2^{\mathbb{R}}$. In some cases, the following property of the operator $\Phi_{\mathcal{J}}$ is very useful. We denote it by IV along to the properties I-III in Proposition 1.8.

IV. For every $\mathcal{S}$-measurable set $X$,

$$
X \sim \Phi_{\mathcal{J}}(X) .
$$

It is an analogue of the classical Lebesgue density theorem in the abstract sense when we consider the density with respect to an invariant $\sigma$-ideal $\mathcal{J}$.

Proposition 1.14 (cf. [1]). The following conditions are equivalent:

1. $\forall X \in S X \backslash \Phi_{\mathcal{J}}(X) \in \mathcal{J}$,

2. $\forall_{X \in S} X \sim \Phi_{\mathcal{J}}(X)$.

By Proposition 1.14, condition IV can be interpreted as: $\mathcal{J}$-almost every point of every $\mathcal{S}$-measurable set is a $\mathcal{J}$-density point of that set.

Definition 1.15. We say that an invariant pair $(\mathcal{S}, \mathcal{J})$ has the $\mathcal{J}$-density property if condition IV is satisfied.

The $\mathcal{J}$-density property for a pair $(S, \mathcal{J})$ implies that for every $X \in S$ we have $\Phi_{\mathcal{J}}(X) \in S$.

Operator $\Phi_{\mathcal{J}}$ satisfying conditions I-IV is called, in the lifting theory, the lower density operator on $(\mathbb{R}, S, \mathcal{J})$. Thus in the context of Proposition 6.37 and Theorem 6.39 from [10] we have

Theorem 1.16. Every invariant pair $(\mathcal{S}, \mathcal{J})$ having the $\mathcal{J}$-density property and satisfying countable chain condition (c.c.c.) generates the $\mathcal{J}$-density topology $\mathcal{T}_{\mathcal{J}}$.

Theorem 1.17. If an invariant pair $(\mathcal{S}, \mathcal{J})$ has the $\mathcal{J}$-density property and generates the $\mathcal{J}$-density topology, then $\mathcal{K}\left(\mathcal{T}_{\mathcal{J}}\right)=\mathcal{J}$ and $\mathcal{B} a\left(\mathcal{T}_{\mathcal{J}}\right)=\mathcal{S}$.

There are two fundamental examples in which, by Theorem 1.16, we get the abstract density topologies. 
Example 1.18. Let $\mathcal{S}=\mathcal{L}$ and $\mathcal{J}=\mathbb{L}$. It is well known that the pair $(\mathcal{S}, \mathcal{J})$ is invariant. Also, $(\mathcal{S}, \mathcal{J})$ satisfies c.c.c. Moreover, for each set $X \in \mathcal{S}$, $\Phi_{\mathcal{J}}(X)$ is the set of density points of $X$. By the Lebesgue density theorem, we have that $X \sim \Phi_{\mathcal{J}}(X)$ and thus, by Theorem 1.16, the family

$$
\mathcal{T}_{\mathcal{J}}=\left\{X \in \mathcal{S}: X \subset \Phi_{\mathcal{J}}(X)\right\}
$$

is a topology known as the density topology, usually labelled by $\mathcal{T}_{d}$ and called the $d$-topology(see [4], [5]).

Example 1.19. Let $\mathcal{S}=B a$ and $\mathcal{J}=\mathbb{K}$. The pair $(\mathcal{S}, \mathcal{J})$ is invariant and satisfies c.c.c. We easily conclude that, for each set $V \in \mathcal{T}_{0}, V \subset \Phi_{\mathcal{J}}(V) \subset \bar{V}$ (see [15]). Since $\bar{V} \backslash V$ is a meager set, we have that $\Phi_{\mathcal{J}}(V) \sim V$. If $X \in \mathcal{S}$, then $X=V \triangle Z$ where $V \in \mathcal{T}_{0}$ and $Z \in \mathcal{J}$. Since $X \sim V$, from Proposition 1.8 we have $\Phi_{\mathcal{J}}(X)=\Phi_{\mathcal{J}}(V)$. This implies that $\Phi_{\mathcal{J}}(X) \sim X$. By Theorem 1.16 , the family

$$
\mathcal{T}_{\mathcal{J}}=\left\{X \in \mathcal{S}: X \subset \Phi_{\mathcal{J}}(X)\right\}
$$

forms a topology. It is a category analogue of the density topology (see [13], $[3]$ ). In the literature on that topic, it is known as the $\mathcal{I}$-density topology. By that reason we shall denote it is the sequel by $\mathcal{I}_{\mathcal{I}}$.

Further examples of the $\mathcal{J}$-density topologies generated by invariant pairs $(\mathcal{S}, \mathcal{J})$ having the $\mathcal{J}$-density property are included in [1]. They concern product $\sigma$-ideals, and $\sigma$-algebras on the plane, related to them.

The $\mathcal{J}$-density property for the pairs $(\mathcal{S}, \mathcal{J})$ in Examples 1.18 and 1.19 plays an important role in deriving the $\mathcal{J}$-density topology by a lower density operator. We consider an example convincing us that the $\mathcal{J}$-density property of the pair $(\mathcal{S}, \mathcal{J})$ is not necessary for the operator $\Phi_{\mathcal{J}}$ to induce the $\mathcal{J}$-density topology.

First, we pay attention to the following

Lemma 1.20. If $\left(\mathcal{S}_{n}, \mathcal{J}_{n}\right)_{n \in \mathbb{N}}$ is a sequence of invariant pairs such that, for every positive integer $n$, the pair $\left(\mathcal{S}_{n}, \mathcal{J}_{n}\right)_{n \in \mathbb{N}}$ induces the $\mathcal{J}$-density topology $\mathcal{T}_{\mathcal{J}_{n}}$, then the pair $(\mathcal{S}, \mathcal{J})$, where $\mathcal{S}=\bigcap_{n=1}^{\infty} \mathcal{S}_{n}$ and $\mathcal{J}=\bigcap_{n=1}^{\infty} \mathcal{J}_{n}$, is invariant and yields the $\mathcal{J}$-density topology $\mathcal{T}_{\mathcal{J}}$. Moreover, $\mathcal{T}_{\mathcal{J}}=\bigcap_{n=1}^{\infty} \mathcal{T}_{\mathcal{J}_{n}}$.

Proof. It is clear that the pair $(\mathcal{S}, \mathcal{J})$ is invariant. To prove that $\mathcal{T}_{\mathcal{J}}=$ $\bigcap_{n=1}^{\infty} \mathcal{T}_{\mathcal{J}_{n}}$, it is sufficient to observe that, for each $X \in \mathcal{S}$, we have

$$
\Phi_{\mathcal{J}}(X)=\bigcap_{n=1}^{\infty} \Phi_{\mathcal{J}_{n}}(X)
$$

For every positive integer $n, \mathcal{J} \subset \mathcal{J}_{n}$. This implies that $\Phi_{\mathcal{J}}(X) \subset$ $\bigcap_{n=1}^{\infty} \Phi_{\mathcal{J}_{n}}(X)$. Now, let $x \in \bigcap_{n=1}^{\infty} \Phi_{\mathcal{J}_{n}}(X)$. We show that $x \in \Phi_{\mathcal{J}}(X)$. 
Let $\left\{n_{i}\right\}_{i \in \mathbb{N}}$ be an arbitrary sequence of positive integers. We prove that there exists a subsequence $\left\{n_{i_{k}}\right\}_{k \in \mathbb{N}}$ such that $\chi_{n_{i_{k}}(X-x) \cap[-1,1]} \underset{k \rightarrow \infty}{\longrightarrow} \chi_{[-1,1]}$ $\mathcal{J}$-a.e. Since $x \in \bigcap_{n=1}^{\infty} \Phi_{\mathcal{J}_{n}}(X)$, we can construct, by induction, a sequence of sequences $\left\{n_{i}^{(m)}\right\}_{i, m \in \mathbb{N}}$ such that, for every $m,\left\{n_{i}^{(m)}\right\}_{i, m \in \mathbb{N}} \subset$ $\left\{n_{i}^{(m-1)}\right\}_{i, m \in \mathbb{N}}$, where $\left\{n_{i}^{(0)}\right\}=\left\{n_{i}\right\}_{i \in \mathbb{N}}$, and a sequence of sets $\left\{A_{m}\right\}_{m \in \mathbb{N}}$ such that $A_{m} \in \mathcal{J}_{m}$ for each positive integer $m$, and that $\chi_{n_{i}^{(m)}(X-x) \cap[-1,1]}(x)$ $\underset{i \rightarrow \infty}{\longrightarrow} \chi_{[-1,1]}(x)$ for any $x \notin A_{m}$. This implies that the sequence $\left\{n_{i_{m}}\right\}_{m \in \mathbb{N}}$, where $n_{i_{m}}=n_{m}^{(m)}$ for each $m \in \mathbb{N}$ (in other words $\left\{n_{i_{m}}\right\}_{m \in \mathbb{N}}$ is the diagonal sequence for the double sequence $\left\{n_{i}^{(m)}\right\}_{i, m \in \mathbb{N}}$ ) has the property that $\chi_{n_{i_{m}}(X-x) \cap[-1,1]}(x) \underset{i \rightarrow \infty}{\longrightarrow} \chi_{[-1,1]}(x)$ for any $x \notin \bigcap_{m=1}^{\infty} A_{m}$. Namely, if $x \notin$ $\bigcap_{m=1}^{\infty} A_{m}$, there exists $m_{0}$ such that $x \notin A_{m_{0}}$. Then $\chi_{n_{i}^{\left(m_{0}\right)}(X-x) \cap[-1,1]}(x)$ $\underset{i \rightarrow \infty}{\longrightarrow} \chi_{[-1,1]}(x)$. Hence the sequence $\left\{\chi_{n_{i_{m}}}(X-x) \cap[-1,1](x)\right\}_{m \in \mathbb{N}}$ converges to $\chi_{[-1,1]}(x)$. Since $\bigcap_{m=1}^{\infty} A_{m} \in \mathcal{J}$, we conclude that $x$ is a $\mathcal{J}$-density point of $X$. Hence $x \in \Phi_{\mathcal{J}}(X)$. Now, we have

$$
\begin{aligned}
\mathcal{T}_{\mathcal{J}} & =\left\{X \in \mathcal{S}: X \subset \Phi_{\mathcal{J}}(X)\right\}=\left\{X \in \bigcap_{n=1}^{\infty} \mathcal{S}_{n}: X \subset \bigcap_{n=1}^{\infty} \Phi_{\mathcal{J}_{n}}(X)\right\} \\
& =\bigcap_{n=1}^{\infty}\left\{X \in \mathcal{S}_{n}: X \subset \Phi_{\mathcal{J}_{n}}(X)\right\}=\bigcap_{n=1}^{\infty} \mathcal{T}_{\mathcal{J}_{n}} .
\end{aligned}
$$

It follows that $\mathcal{T}_{\mathcal{J}}$ is a topology as the intersection of topologies and, at the same time, $\mathcal{T}_{\mathcal{J}}=\bigcap_{n=1}^{\infty} \mathcal{T}_{\mathcal{J}_{n}}$.

Example 1.21. Let $\mathcal{S}=\mathcal{B} a \cap \mathcal{L}$ and $\mathcal{J}=\mathbb{K} \cap \mathbb{L}$. The pair $(\mathcal{S}, \mathcal{J})$ is invariant. By Examples 1.18, 1.19 and Lemma 1.20 the pair $(\mathcal{S}, \mathcal{J})$ generates the $\mathcal{J}$-density topology $\mathcal{T}_{\mathcal{J}}$ for which $\mathcal{T}_{\mathcal{J}}=\mathcal{T}_{d} \cap \mathcal{T}_{\mathcal{I}}$. We point out that the pair $(\mathcal{S}, \mathcal{J})$ does not possess the $\mathcal{J}$-density property. Namely, let Borel sets $A$ and $B$ be a decomposition of reals, such that $A \in \mathbb{L}, B \in \mathbb{K}$ (see [12]). Then $A \in \mathcal{S}$ and $A \notin \mathcal{J}$. By Lemma 1.20, we have $\Phi_{\mathcal{J}}(A)=\Phi_{\mathbb{L}}(A) \cap \Phi_{\mathbb{K}}(A)$. Since $\Phi_{\mathbb{L}}(A)=\emptyset$, we have that $\Phi_{\mathcal{J}}(A)=\emptyset$. Consequently, $\Phi_{\mathcal{J}}(X) \sim X$ for each $X \in \mathcal{S}$.

It is also true in this example that:

Lemma 1.22 (cf. [2]). $\mathcal{B} a \cap \mathcal{L}=\mathcal{B} \triangle(\mathbb{K} \cap \mathbb{L})$.

This example shows that the $\mathcal{J}$-density property is not necessary to assert that an invariant pair $(\mathcal{S}, \mathcal{J})$ yields the $\mathcal{J}$-density topology. This is a motivation for considering the $\mathcal{J}$-density topology related to an invariant pair $(\mathcal{S}, \mathcal{J})$ without the $\mathcal{J}$-density property. 
We have the following

Observation 1.23. For every invariant $\sigma$-ideal $\mathcal{J}$, there exists the smallest $\sigma$-algebra $\mathcal{S}(\mathcal{J})$ such that $(\mathcal{S}(\mathcal{J}), \mathcal{J})$ is an invariant pair generating the $\mathcal{J}$-density topology.

Proof. Let $\left\{\mathcal{S}_{t}\right\}_{t \in T}$ be the family of all invariant $\sigma$-algebras such that, for each $t \in T$, the pair $\left(\mathcal{S}_{t}, \mathcal{J}\right)$ is invariant and yields the $\mathcal{J}$-density topology $\mathcal{T}_{\mathcal{J}}^{t}$. We see that $T \neq \emptyset$ because, by Example 1.13 , the pair $\left(2^{\mathbb{R}}, \mathcal{J}\right)$ is invariant and yields the $\mathcal{J}$-density topology. Putting $\mathcal{S}(\mathcal{J})=\bigcap_{t \in T} \mathcal{S}_{t}$, we have that the pair $(\mathcal{S}(\mathcal{J}), \mathcal{J})$ is invariant and

$$
\begin{aligned}
\mathcal{T}_{\mathcal{J}} & =\left\{X \in \mathcal{S}(\mathcal{J}): X \subset \Phi_{\mathcal{J}}(X)\right\} \\
& =\bigcap_{t \in T}\left\{X \in \mathcal{S}_{t}: X \subset \Phi_{\mathcal{J}}(X)\right\}=\bigcap_{t \in T} \mathcal{T}_{\mathcal{J}}^{t} .
\end{aligned}
$$

The last assertion means that the pair $(\mathcal{S}(\mathcal{J}), \mathcal{J})$ induces the $\mathcal{J}$-density topology.

Remark 1.24. By the definition of the invariant pair $(\mathcal{S}(\mathcal{J}), \mathcal{J})$, it is clear that

$$
\mathcal{B} \triangle \mathcal{J} \subset \mathcal{S}(\mathcal{J}) \subset 2^{\mathbb{R}}
$$

In Examples 1.18 and 1.19 we see that if $\mathcal{J}=\mathbb{L}$ or $\mathcal{J}=\mathbb{K}$, then $\mathcal{S}(\mathcal{J})=$ $\mathcal{B} \triangle \mathcal{J}$. Also, for $\mathcal{J}=\mathbb{K} \cap \mathbb{L}$, from Example 1.21 and Lemma 1.22 we have $\mathcal{S}(\mathcal{J})=\mathcal{B} \Delta \mathcal{J}$. However, Example 1.11 says that if $\mathcal{J}$ is the $\sigma$-ideal of countable sets, then $\mathcal{S}(\mathcal{J}) \neq \mathcal{B}=\mathcal{B} \triangle \mathcal{J}$. Simultaneously, $\mathcal{S}(\mathcal{J}) \subset \mathcal{B} \triangle$ $(\mathbb{K} \cap \mathbb{L})$. Thus $\mathcal{S}(\mathcal{J}) \neq 2^{\mathbb{R}}$.

Problem 1.25. Does there exist an invariant $\sigma$-ideal $\mathcal{J}$ such that $\mathcal{S}(\mathcal{J})=$ $2^{\mathbb{R}}$ ?

\section{Properties of the density topologies}

In the definition of the $\mathcal{J}$-density topology $\mathcal{T}_{\mathcal{J}}$ generated by an invariant pair $(\mathcal{S}, \mathcal{J})$, only some $\mathcal{S}$-measurable sets are taken under consideration: namely, an $\mathcal{S}$-measurable set $X$ is $\mathcal{T}_{\mathcal{J}}$-open if $X \subset \Phi_{\mathcal{J}}(X)$. Other $\mathcal{S}$-measurable sets are not members of the family $\mathcal{T}_{\mathcal{J}}$. In this context, the natural question arises:

How can we decrease the $\sigma$-algebra $\mathcal{S}$ in the sense of inclusion to another $\sigma$-algebra $\mathcal{S}^{\prime} \subset \mathcal{S}$ such that the pair $\left(\mathcal{S}^{\prime}, \mathcal{J}\right)$ is invariant and yields the $\mathcal{J}$-density topology $\mathcal{T}_{\mathcal{J}}^{\prime}$ which is identical with the $\mathcal{J}$-density topology $\mathcal{T}_{\mathcal{J}}$ ? 
Theorem 2.1. Let $(\mathcal{S}, \mathcal{J})$ be an invariant pair generating the $\mathcal{J}$-density topology $\mathcal{T}_{\mathcal{J}}$. The family $\mathcal{K}\left(\mathcal{T}_{\mathcal{J}}\right)$ of meager sets with respect to the topology $\mathcal{T}_{\mathcal{J}}$ is identical with $\mathcal{J}$ if and only if there exists a $\sigma$-algebra $\mathcal{S}^{\prime}$ such that

1. $\mathcal{J} \subset \mathcal{S}^{\prime} \subset \mathcal{S}$,

2. $\left(\mathcal{S}^{\prime}, \mathcal{J}\right)$ is invariant,

3. $\left(\mathcal{S}^{\prime}, \mathcal{J}\right)$ has the $\mathcal{J}$-density property,

4. $\mathcal{T}_{\mathcal{J}}^{\prime}=\left\{X \in \mathcal{S}^{\prime}: X \subset \Phi_{\mathcal{J}}(X)\right\}$ is the $\mathcal{J}$-density topology associated with the pair $\left(\mathcal{S}^{\prime}, \mathcal{J}\right)$, and $\mathcal{T}_{\mathcal{J}}^{\prime}=\mathcal{T}_{\mathcal{J}}$.

Proof. Necessity. Let $\mathcal{S}^{\prime}=\mathcal{T}_{\mathcal{J}} \triangle \mathcal{J}$. Since $\mathcal{J}=\mathcal{K}\left(\mathcal{T}_{\mathcal{J}}\right)$, we have that $\mathcal{S}^{\prime}$ is the $\sigma$-algebra of all sets having the Baire property with respect to the topology $\mathcal{T}_{\mathcal{J}}$. Because $\mathcal{J} \subset \mathcal{S}$ and $\mathcal{T}_{\mathcal{J}} \subset \mathcal{S}$, we see that condition 1 is satisfied. By Proposition 1.9, we see that the family $\mathcal{T}_{\mathcal{J}}$ is invariant with respect to every linear operation of the form $n x+a$ where $n$ is a positive integer and $a$ is an arbitrary real number. It implies that the pair $\left(\mathcal{S}^{\prime}, \mathcal{J}\right)$ is invariant. Now, we prove that the pair $\left(\mathcal{S}^{\prime}, \mathcal{J}\right)$ has the $\mathcal{J}$-density property. Let $X \in \mathcal{S}^{\prime}$. Then $X=V \triangle Y$ where $V \in \mathcal{T}_{\mathcal{J}}$ and $Y \in \mathcal{J}$. Thus $\Phi_{\mathcal{J}}(X)=$ $\Phi_{\mathcal{J}}(V \Delta Y)=\Phi_{\mathcal{J}}(V) \supset V$. Hence $X \backslash \Phi_{\mathcal{J}}(X) \subset(V \triangle Y) \backslash V \subset Y \in \mathcal{J}$. Since $\mathcal{S}^{\prime}$ is a $\sigma$-algebra, we conclude, by Proposition 1.14 that $X \sim \Phi_{\mathcal{J}}(X)$ for any $X \in \mathcal{S}^{\prime}$. Hence the pair $\left(\mathcal{S}^{\prime}, \mathcal{J}\right)$ has the $\mathcal{J}$-density property. Further, we prove condition 4 . It is sufficient to establish that $\mathcal{T}_{\mathcal{J}}^{\prime}=\mathcal{T}_{\mathcal{J}}$. Since $\mathcal{S}^{\prime} \subset \mathcal{S}$, we have that $\mathcal{T}_{\mathcal{J}}^{\prime} \subset \mathcal{T}_{\mathcal{J}}$. The inclusion $\mathcal{T}_{\mathcal{J}} \subset \mathcal{S}^{\prime}$ implies $\mathcal{T}_{\mathcal{J}} \subset \mathcal{T}_{\mathcal{J}}^{\prime}$. Thus we conclude that $\mathcal{T}_{\mathcal{J}}^{\prime}$ is a topology and, by the definition of the family $\mathcal{T}_{\mathcal{J}}^{\prime}$, we see that it is the $\mathcal{J}$-density topology associated with the pair $\left(\mathcal{S}^{\prime}, \mathcal{J}\right)$.

Sufficiency. Let us consider the pair $\left(\mathcal{S}^{\prime}, \mathcal{J}\right)$ satisfying conditions $1-4$. By condition 2 , we can define the family $\mathcal{T}_{\mathcal{J}}^{\prime}$ with respect to the pair $\left(\mathcal{S}^{\prime}, \mathcal{J}\right)$. Condition 4 guarantees that $\mathcal{T}_{\mathcal{J}}^{\prime}$ is the $\mathcal{J}$-density topology associated with the pair $\left(\mathcal{S}^{\prime}, \mathcal{J}\right)$. Condition 3 implies that the topology $\mathcal{T}_{\mathcal{J}}^{\prime}$ is induced by the lower operator $\Phi_{\mathcal{J}}$ and thus, by Theorem 1.17 the family $\mathcal{K}\left(\mathcal{T}_{\mathcal{J}}^{\prime}\right)$ of meager sets with respect to the topology $\mathcal{T}_{\mathcal{J}}^{\prime}$ is identical with the $\sigma$-ideal $\mathcal{J}$. The equality $\mathcal{T}_{\mathcal{J}}^{\prime}=\mathcal{T}_{\mathcal{J}}$ implies that $\mathcal{K}\left(\mathcal{T}_{\mathcal{J}}^{\prime}\right)=\mathcal{J}$.

Remark 2.2. There exists an example of an invariant pair $(\mathcal{S}, \mathcal{J})$ without the $\mathcal{J}$-density property for which there exists a $\sigma$-algebra $\mathcal{S}^{\prime} \subset \mathcal{S}$ such that the pair $\left(\mathcal{S}^{\prime}, \mathcal{J}\right)$ is invariant and has the $\mathcal{J}$-density property. This example is based on an extension of Lebesgue measure (see [6], [8]).

Proposition 2.3. If $(\mathcal{S}, \mathcal{J})$ is an invariant pair generating the $\mathcal{J}$-density topology $\mathcal{T}_{\mathcal{J}}$, such that $\mathcal{K}\left(\mathcal{T}_{\mathcal{J}}\right)=\mathcal{J}$, then the smallest $\sigma$-algebra $\mathcal{S}(\mathcal{J})$ such that the invariant pair $(\mathcal{S}(\mathcal{J}), \mathcal{J})$ generates the $\mathcal{J}$-density topology identical with $\mathcal{T}_{\mathcal{J}}$ is equal to $\mathcal{B} a\left(\mathcal{T}_{\mathcal{J}}\right)$. 
Proof. By the proof of Theorem 2.1, we conclude that $\mathcal{S}(\mathcal{J}) \subset \mathcal{T}_{\mathcal{J}} \triangle \mathcal{J}$. Since $\mathcal{T}_{\mathcal{J}} \subset \mathcal{S}(\mathcal{J})$ and $\mathcal{J} \subset \mathcal{S}(\mathcal{J})$, we have that $\mathcal{T}_{\mathcal{J}} \triangle \mathcal{J} \subset \mathcal{S}(\mathcal{J})$. Thus $\mathcal{S}(\mathcal{J})=\mathcal{T}_{\mathcal{J}} \triangle \mathcal{J}=\mathcal{T}_{\mathcal{J}} \triangle \mathcal{K}\left(\mathcal{T}_{\mathcal{J}}\right)=\mathcal{B} a\left(\mathcal{T}_{\mathcal{J}}\right)$

Proposition 2.4. If $(\mathcal{S}, \mathcal{J})$ is an invariant pair generating the $\mathcal{J}$-density topology $\mathcal{T}_{\mathcal{J}}$, then

1. $\mathcal{B} \Delta \mathcal{J} \subset \mathcal{T}_{\mathcal{J}} \triangle \mathcal{K}\left(\mathcal{T}_{\mathcal{J}}\right)$,

2. $\mathcal{B} \triangle \mathcal{J} \subset \mathcal{S}(\mathcal{J}) \subset \mathcal{S}$.

Moreover, if the pair $(\mathcal{S}, \mathcal{J})$ has the $\mathcal{J}$-density property, then $\mathcal{T}_{\mathcal{J}} \triangle \mathcal{K}\left(\mathcal{T}_{\mathcal{J}}\right)=$ $\mathcal{S}(\mathcal{J})=\mathcal{S}$.

Proof. The above inclusions are obvious. If the pair $(\mathcal{S}, \mathcal{J})$ has the $\mathcal{J}$ density property, then, by Theorem 1.17 , we have $\mathcal{K}\left(\mathcal{T}_{\mathcal{J}}\right)=\mathcal{J}$ and $\mathcal{S}=$ $\mathcal{T}_{\mathcal{J}} \triangle \mathcal{K}\left(\mathcal{T}_{\mathcal{J}}\right)$. Thus, by the previous proposition, the equality holds.

Corollary 2.5. If $(\mathcal{B} \triangle \mathcal{J}, \mathcal{J})$ is an invariant pair generating the $\mathcal{J}$-density topology $\mathcal{T}_{\mathcal{J}}$ and $(\mathcal{B} \triangle \mathcal{J}, \mathcal{J})$ has the $\mathcal{J}$-density property, then $\mathcal{B} a\left(\mathcal{T}_{\mathcal{J}}\right)=$ $\mathcal{S}(\mathcal{J})=\mathcal{B} \triangle \mathcal{J}$

Now, we estimate the cardinality of $S(\mathcal{J})$. We need the following lemmas:

Lemma 2.6. For each $X \subset \mathbb{R}$, we have $\Phi_{\mathcal{J}_{0}}(X) \subset X$.

Proof. Let $x \in \Phi_{\mathcal{J}_{0}}(X)$. Thus 0 is a $\mathcal{J}_{0}$-density point of the set $X-x$. From Lemma 1.5 we easily conclude that $0 \in X-x$. Thus $x \in X$.

Lemma 2.7. There exists a nonempty perfect set $F \subset \mathbb{R}$ such that $\Phi_{\mathcal{J}_{0}}((\mathbb{R} \backslash F) \cup\{x\})=(\mathbb{R} \backslash F) \cup\{x\}$ for each $x \in F$.

Proof. Let $H$ be any Hamel basis of the space of reals over the field of rational numbers, containing a nonempty perfect set $F$ (see [9]). Since $\mathbb{R} \backslash F \in \mathcal{T}_{0}$, Proposition 1.10 gives that $\mathbb{R} \backslash F \subset \Phi_{\mathcal{J}_{0}}(\mathbb{R} \backslash F)$. We have to prove that each point $x \in F$ is a $\mathcal{J}_{0}$-density point of $(\mathbb{R} \backslash F) \cup\{x\}$. Let $\left\{n_{k}\right\}_{k \in \mathbb{N}}$ be any increasing subsequence of positive integers. We show that

$$
[-1,1] \subset \bigcup_{j=1}^{\infty} \bigcap_{k=j}^{\infty} n_{k}(((\mathbb{R} \backslash F) \cup\{x\})-x) .
$$

Let $\alpha \in[-1,1]$. Clearly, we may assume that $\alpha \neq 0$. There exists at most one positive integer $k$ such that $\alpha \notin n_{k}((\mathbb{R} \backslash F)-x)$. Indeed, let us 
suppose that we have $k_{1}$ and $k_{2}$ such that $k_{1} \neq k_{2}$ and $\alpha \notin n_{k_{1}}((\mathbb{R} \backslash F)-x)$, $\alpha \notin n_{k_{2}}((\mathbb{R} \backslash F)-x)$. Consequently,

$$
\frac{\alpha}{n_{k_{1}}}+x=z_{1} \text { and } \frac{\alpha}{n_{k_{2}}}+x=z_{2}, \quad \text { where } z_{1}, z_{2} \in F \text {. }
$$

Since $\alpha \neq 0$, we have $z_{1} \neq z_{2} \neq x$ and

$$
\left(n_{k_{1}}-n_{k_{2}}\right) x+n_{k_{1}} z_{1}+n_{k_{2}} z_{2}=0 .
$$

Since $H$ is a Hamel basis, $n_{k_{1}}=n_{k_{2}}=0$, contrary to the fact that $n_{k_{1}} \neq n_{k_{2}}$ and, consequently, $\alpha \in \bigcup_{l=1}^{\infty} \bigcap_{k=l}^{\infty} n_{k}(((\mathbb{R} \backslash F) \cup\{x\})-x)$. Therefore $(\mathbb{R} \backslash F) \cup$ $\{x\} \subset \Phi_{\mathcal{J}_{0}}((\mathbb{R} \backslash F) \cup\{x\})$. By the previous lemma, we have $\Phi_{\mathcal{J}_{0}}((\mathbb{R} \backslash F) \cup$ $\{x\})=(\mathbb{R} \backslash F) \cup\{x\}$.

Theorem 2.8. If $\mathcal{T}_{\mathcal{J}}$ is the family associated with the invariant pair $(\mathcal{S}, \mathcal{J})$, then $\mathcal{T}_{\mathcal{J}} \backslash \mathcal{T}_{0} \neq \emptyset$.

Proof. By Lemma 2.7, there exists a nonempty perfect set $F \subset \mathbb{R}$ such that $\Phi_{\mathcal{J}_{0}}((\mathbb{R} \backslash F) \cup\{x\})=(\mathbb{R} \backslash F) \cup\{x\}$ for each $x \in F$. Let $x \in F$ and $Y=(\mathbb{R} \backslash F) \cup\{x\}$, then $Y \in \mathcal{B}$. Thus $Y \in \mathcal{S}$ and $Y=\Phi_{\mathcal{J}_{0}}(Y) \subset \Phi_{\mathcal{J}}(Y)$. Hence $Y \in \mathcal{T}_{\mathcal{J}} \backslash \mathcal{T}_{0}$.

Theorem 2.9. For every invariant pair $(\mathcal{S}, \mathcal{J})$ generating the $\mathcal{J}$-density topology $\mathcal{T}_{\mathcal{J}}$, card $\mathcal{S}=2^{\mathfrak{c}}$.

Proof. By Lemma 2.7 there exists a nonempty perfect set $F \subset \mathbb{R}$ such that, for each $x \in F$, we have $\Phi_{\mathcal{J}_{0}}((\mathbb{R} \backslash F) \cup\{x\})=(\mathbb{R} \backslash F) \cup\{x\}$. It is clear that $\Phi_{\mathcal{J}_{0}}((\mathbb{R} \backslash F) \cup\{x\}) \subset \Phi_{\mathcal{J}}((\mathbb{R} \backslash F) \cup\{x\})$. Since $(\mathbb{R} \backslash F) \cup\{x\} \in \mathcal{S}$, we conclude that $(\mathbb{R} \backslash F) \cup\{x\} \in \mathcal{T}_{\mathcal{J}}$ for each $x \in F$. Let us suppose that card $\mathcal{S}<2^{\mathfrak{c}}$. Then there exists a set $X \subset F$ such that $(\mathbb{R} \backslash F) \cup X \notin \mathcal{S}$. At the same time, $(\mathbb{R} \backslash F) \cup X=\bigcup_{x \in X}((\mathbb{R} \backslash F) \cup\{x\}) \in \mathcal{T}_{\mathcal{J}}$ and, by the definition of the $\mathcal{J}$-density topology, it should be a member of $\mathcal{S}$. This contradiction proves that $\operatorname{card} \mathcal{S}=2^{\mathfrak{c}}$.

Corollary 2.10. For every invariant $\sigma$-ideal $\mathcal{J}$, card $\mathcal{S}(\mathcal{J})=2^{\mathfrak{c}}$.

Now we present some properties of the density topologies with respect to $\sigma$-ideals having some connections with measure and category.

Definition 2.11. We shall say that a $\sigma$-ideal $\mathcal{J} \subset 2^{\mathbb{R}}$ is controlled by measure if $\mathcal{J} \subset \mathbb{L}$ or $\mathbb{L} \subset \mathcal{J}$.

Definition 2.12. We shall say that a $\sigma$-ideal $\mathcal{J} \subset 2^{\mathbb{R}}$ is controlled by category if $\mathcal{J} \subset \mathbb{K}$ or $\mathbb{K} \subset \mathcal{J}$. 
The following lemma will be useful in further considerations.

Lemma 2.13. If $\left(\mathcal{S}_{1}, \mathcal{J}_{1}\right)$ and $\left(\mathcal{S}_{2}, \mathcal{J}_{2}\right)$ are invariant pairs generating the $\mathcal{J}_{1}$-density topology $\mathcal{T}_{\mathcal{J}_{1}}$ and the $\mathcal{J}_{2}$-density topology $\mathcal{T}_{\mathcal{J}_{2}}$, respectively, and $\mathcal{S}_{1} \subset \mathcal{S}_{2}, \mathcal{J}_{1} \subset \mathcal{J}_{2}$, then the pair $\left(\mathcal{S}_{2}, \mathcal{J}_{1}\right)$ is invariant and generates the $\mathcal{J}_{1}$-density topology $\mathcal{T}_{\mathcal{J}_{1}}^{2}$ for which $\mathcal{T}_{\mathcal{J}_{1}} \subset \mathcal{T}_{\mathcal{J}_{1}}^{2} \subset \mathcal{T}_{\mathcal{J}_{2}}$.

Proof. It is obvious that the pair $\left(\mathcal{S}_{2}, \mathcal{J}_{1}\right)$ is invariant. Let $\mathcal{T}_{\mathcal{J}_{1}}^{2}=\{X \in$ $\left.\mathcal{S}_{2}: X \subset \Phi_{\mathcal{J}_{\infty}}(X)\right\}$. By Proposition 1.9, it is sufficient to show that the union of any subfamily of sets belonging to the family $\mathcal{T}_{\mathcal{J}_{1}}^{2}$ is a member of $\mathcal{T}_{\mathcal{J}_{1}}^{2}$. Since $\mathcal{J}_{1} \subset \mathcal{J}_{2}$, therefore $\mathcal{T}_{\mathcal{J}_{1}}^{2} \subset \mathcal{T}_{\mathcal{J}_{2}}$. Hence the union of any subfamily of subsets of the family $\mathcal{T}_{\mathcal{J}_{1}}^{2}$ is a $\mathcal{T}_{\mathcal{J}_{2}}$-open set. Thus it is an $\mathcal{S}_{2}$-measurable set and, in that way, belongs to the family $\mathcal{T}_{\mathcal{J}_{1}}^{2}$. Since $\mathcal{S}_{1} \subset \mathcal{S}_{2}$, we have $\mathcal{T}_{\mathcal{J}_{1}} \subset \mathcal{T}_{\mathcal{J}_{1}}^{2}$

Theorem 2.14. If $\mathcal{J}$ is an invariant $\sigma$-ideal such that $\mathcal{J} \subset \mathbb{K}$, then the $\mathcal{J}$-density topology $\mathcal{T}_{\mathcal{J}}$ generated by the pair $(\mathcal{S}(\mathcal{J}), \mathcal{J})$ has the property that $\mathcal{K}\left(\mathcal{T}_{\mathcal{J}}\right)=\mathbb{K}$ and $\mathcal{B} a\left(\mathcal{T}_{\mathcal{J}}\right)=\mathcal{B} a$

Proof. We show that $\mathcal{K}\left(\mathcal{T}_{\mathcal{J}}\right) \subset \mathbb{K}$. Let $X \in \mathcal{K}\left(\mathcal{T}_{\mathcal{J}}\right)$. It suffices to assume that a $X$ is a $\mathcal{T}_{\mathcal{J}}$-nowhere dense closed set. It is clear that $X \in \mathcal{S}(\mathcal{J})$. It is obvious that the pair $(\mathcal{B} a, \mathcal{J})$ is invariant. From Example 1.21 and Lemma 2.13 we conclude that this pair generates the $\mathcal{J}$-density topology $\mathcal{T}_{\mathcal{J}}^{\prime}$, and $\mathcal{T}_{\mathcal{J}} \subset \mathcal{I}_{\mathcal{J}}^{\prime} \subset \mathcal{I}_{\mathcal{I}}$. This implies that $\mathbb{R} \backslash X \in \mathcal{I}_{\mathcal{I}}$ and then $X \in \mathcal{B} a$. The set $X$ having the Baire property has the form $X=V \triangle Z$, where $V \in \mathcal{T}_{0}$ and $Z \in \mathbb{K}$. We show that $V=\emptyset$. Let us suppose that $V \neq \emptyset$. Of course, $V \in \mathcal{T}_{\mathcal{J}}$. Since $X$ is $\mathcal{T}_{\mathcal{J}}$-nowhere dense, there exists a nonempty $\mathcal{T}_{\mathcal{J}}$-open set $V_{1}$ such that $V_{1} \subset V$ and $V_{1} \cap X=\emptyset$. Since $\mathcal{T}_{\mathcal{J}} \subset \mathcal{I}_{\mathcal{I}}$, we have $V_{1} \in \mathcal{T}_{\mathcal{I}}$. As $V_{1} \neq \emptyset$, we infer that $V_{1} \notin \mathbb{K}$. Since $Z=X \Delta V=X \Delta\left[\left(V \backslash V_{1}\right) \cup V_{1}\right] \supset V_{1}$, we get a contradiction with the fact that $Z \in \mathbb{K}$ and $V_{1} \notin \mathbb{K}$. Finally, $V=\emptyset$ and $X=Z$. Therefore $X \in \mathbb{K}$. Now, we show that $\mathbb{K} \subset \mathcal{K}\left(\mathcal{T}_{\mathcal{J}}\right)$. Let $X$ be a nowhere dense set with respect to the natural topology. Assume that $X$ is closed. It is clear that $X$ has the Baire property with respect to $\mathcal{T}_{\mathcal{J}}$. Thus $X=V \triangle Z$, where $V \in \mathcal{T}_{\mathcal{J}} \subset \mathcal{T}_{\mathcal{I}}$ and $Z \in \mathcal{K}\left(\mathcal{T}_{\mathcal{J}}\right) \subset \mathbb{K}$. We have $V=X \triangle Z$, hence $V \in \mathbb{K}$. So, the set $V$ as $\mathcal{T}_{\mathcal{I}}$-open must be empty. This implies that $X=Z$. Consequently, $X \in \mathcal{K}\left(\mathcal{T}_{\mathcal{J}}\right)$. We show that $\mathcal{B} a\left(\mathcal{T}_{\mathcal{J}}\right)=\mathcal{B} a$. By Proposition 1.10 , we have that $\mathcal{T}_{0} \subset \mathcal{T}_{\mathcal{J}}$ and by the first part of the proof that $\mathcal{K}\left(\mathcal{T}_{\mathcal{J}}\right)=\mathbb{K}$, we infer that $\mathcal{B} a \subset \mathcal{B} a\left(\mathcal{T}_{\mathcal{J}}\right)$. We have observed that $\mathcal{S}(\mathcal{J}) \subset \mathcal{B} a$, then $\mathcal{T}_{\mathcal{J}} \subset \mathcal{B} a$. Including the fact that $\mathcal{K}\left(\mathcal{T}_{\mathcal{J}}\right)=\mathbb{K}$ we get that $\mathcal{B} a\left(\mathcal{T}_{\mathcal{J}}\right) \subset \mathcal{B} a$. Finally, $\mathcal{B} a\left(\mathcal{T}_{\mathcal{J}}\right)=\mathcal{B} a$. 
Corollary 2.15. If $\mathcal{S}=\mathcal{B} a \cap \mathcal{L}$ and $\mathcal{J}=\mathbb{K} \cap \mathbb{L}$, then $\mathcal{K}\left(\mathcal{T}_{\mathcal{J}}\right)=\mathbb{K}$ and $\mathcal{B} a\left(\mathcal{T}_{\mathcal{J}}\right)=\mathcal{B} a$.

Proof. By Lemma 1.22 and Remark $1.24, \mathcal{S}(\mathcal{J})=\mathcal{B} a \cap \mathcal{L}$. Thus, by Theorem $2.14, \mathcal{K}\left(\mathcal{T}_{\mathcal{J}}\right)=\mathbb{K}$ and $\mathcal{B} a\left(\mathcal{T}_{\mathcal{J}}\right)=\mathcal{B} a$.

Property 2.16. No invariant pair $(\mathcal{S}, \mathcal{J})$ generating the $\mathcal{J}$-density topology $\mathcal{T}_{\mathcal{J}}$ and such that $\mathcal{J} \varsubsetneqq \mathbb{K}$ possesses the $\mathcal{J}$-density property.

Proof. By Theorem 2.14, the $\mathcal{J}$-density topology $\mathcal{T}_{\mathcal{J}}$ generated by the pair $(\mathcal{S}(\mathcal{J}), \mathcal{J})$ does not possess the $\mathcal{J}$-density property since, otherwise, by Theorem 1.17, we would have that $\mathcal{K}\left(\mathcal{T}_{\mathcal{J}}\right)=\mathcal{J}$, contrary to the fact that $\mathcal{J} \neq \mathbb{K}$. Since $\mathcal{S}(\mathcal{J}) \subset \mathcal{S}$, we deduce that $(\mathcal{S}, \mathcal{J})$ does not possess the $\mathcal{J}$-density property.

It is worth observing that the property described in Theorem 2.14 does not hold in the case of the $\sigma$-ideal $\mathbb{L}$ considered instead of $\mathbb{K}$. Indeed, let $\mathcal{S}=\mathcal{B} a \cap \mathcal{L}$ and $\mathcal{J}=\mathbb{K} \cap \mathbb{L}$. Then, by Corollary 2.15, we have that $\mathcal{K}\left(\mathcal{T}_{\mathcal{J}}\right)=\mathbb{K}$. Hence $\mathcal{K}\left(\mathcal{T}_{\mathcal{J}}\right) \backslash \mathbb{L} \neq \emptyset$ and $\mathbb{L} \backslash \mathcal{K}\left(\mathcal{T}_{\mathcal{J}}\right) \neq \emptyset$.

For invariant $\sigma$-ideals containing $\mathbb{L}$ or $\mathbb{K}$, we have the following

Theorem 2.17. If $\mathcal{J}$ is an invariant $\sigma$-ideal such that $\mathcal{J} \supset \mathbb{K}(\mathcal{J} \supset \mathbb{L})$, then

1. $\mathcal{S}(\mathcal{J})=\mathcal{B} \triangle \mathcal{J}$

2. $(\mathcal{S}(\mathcal{J}), \mathcal{J})$ has the $\mathcal{J}$-density property,

3. $\mathcal{J}=\mathbb{K}(\mathcal{J}=\mathbb{L})$ if and only if $\mathcal{T}_{\mathcal{J}}=\mathcal{T}_{\mathcal{I}}\left(\mathcal{T}_{\mathcal{J}}=\mathcal{T}_{d}\right)$,

where $\mathcal{T}_{\mathcal{J}}$ is the topology generated by the invariant pair $(\mathcal{S}(\mathcal{J}), \mathcal{J})$.

Proof. Let us suppose that $\mathcal{J} \supset \mathbb{K}$. In the case of condition 1, it is sufficient to prove that the invariant pair $(\mathcal{B} \triangle \mathcal{J}, \mathcal{J})$ yields the $\mathcal{J}$-density topology. First of all, we notice that the pair $(\mathcal{B} \triangle \mathcal{J}, \mathcal{J})$ has the $\mathcal{J}$-density property. Namely, let $X \in \mathcal{B} \triangle \mathcal{J}$; then $X=Y \triangle Z$, where $Y \in \mathcal{B}$ and $Z \in \mathcal{J}$. Thus

$$
\begin{aligned}
& X \backslash \Phi_{\mathcal{J}}(X)=(Y \triangle Z) \backslash \Phi_{\mathcal{J}}(Y \triangle Z) \\
& =(Y \triangle Z) \backslash \Phi_{\mathcal{J}}(Y) \subset(Y \triangle Z) \backslash \Phi_{\mathbb{K}}(Y) \subset\left(Y \backslash \Phi_{\mathbb{K}}(Y)\right) \cup Z \in \mathcal{J} .
\end{aligned}
$$

Hence, by Proposition 1.14, for each $X \in \mathcal{B} \triangle \mathcal{J}$, we have $X \sim \Phi_{\mathcal{J}}(X)$. Thus, by Proposition 1.8, the operator $\Phi_{\mathcal{J}}$ is a lower density operator. Moreover, we prove that the pair $(\mathcal{B} \Delta \mathcal{J}, \mathcal{J})$ satisfies countable chain condition (c.c.c.). In fact, it is clear that the pair $(\mathcal{B}, \mathbb{K})$ satisfies c.c.c. Let us suppose that the pair $(\mathcal{B} \triangle \mathcal{J}, \mathcal{J})$ does not satisfy c.c.c. Then there exists a sequence $\left\{X_{\alpha}\right\}_{\alpha<\omega_{1}}$ of pairwise disjoint sets such that, for each $\alpha<\omega_{1}, X_{\alpha}=Y_{\alpha} \triangle Z_{\alpha}$, where $Y_{\alpha} \in \mathcal{B}, Z_{\alpha} \in \mathcal{J}$ and $X_{\alpha} \in(\mathcal{B} \triangle \mathcal{J}) \backslash \mathcal{J}$. We 
put $W_{0}=Y_{0}$ and $W_{\alpha}=Y_{\alpha} \backslash \bigcup_{\beta<\alpha} W_{\beta}$ for any $0<\alpha<\omega_{1}$. If $\alpha_{1}, \alpha_{2}<\omega_{1}$, and $\alpha_{1} \neq \alpha_{2}$, then $W_{\alpha_{1}} \cap W_{\alpha_{2}}=\emptyset$. Since $W_{\alpha} \in \mathcal{B} \backslash \mathcal{J}$ for $0 \leq \alpha<\omega_{1}$, this contradicts the fact that the pair $(\mathcal{B}, \mathbb{K})$ satisfies c.c.c. Now, by Theorem 1.16 , we deduce that the pair $(\mathcal{B} \triangle \mathcal{J}, \mathcal{J})$ yields the $\mathcal{J}$-density topology. In that way, $\mathcal{S}(\mathcal{J})=\mathcal{B} \Delta \mathcal{J}$. The proof of condition 1 is completed. We see that it contains a proof of the fact that the pair $(\mathcal{S}(\mathcal{J}), \mathcal{J})$ has the $\mathcal{J}$-density property.

Now, we prove condition 3. Necessity is obvious. Let us show sufficiency. We only need to prove that $\mathcal{J} \subset \mathbb{K}$. Suppose that $\mathcal{J} \backslash \mathbb{K} \neq \emptyset$. Let $X \in \mathcal{J} \backslash \mathbb{K}$. We consider two cases: $X \in \mathcal{B} \triangle \mathbb{K}$ and $X \notin \mathcal{B} \triangle \mathbb{K}$. If $X \in \mathcal{B} \triangle \mathbb{K}$, then $\Phi_{\mathbb{K}}(X) \cap X \in \mathcal{T}_{\mathcal{I}}$ and $\Phi_{\mathbb{K}}(X) \cap X \neq \emptyset$ because $X \notin \mathbb{K}$. According to the assumption, we have that $\Phi_{\mathbb{K}}(X) \cap X \subset \Phi_{\mathcal{J}}\left(\Phi_{\mathbb{K}}(X) \cap X\right)$. The last assertion is not true because $\Phi_{\mathcal{J}}\left(\Phi_{\mathbb{K}}(X) \cap X\right)=\emptyset$. Let $X \notin \mathcal{B} \triangle \mathbb{K}$. Since $X \in \mathcal{J}$, then $\mathbb{R} \backslash X \in \mathcal{T}_{\mathcal{J}}$. Thus $\mathbb{R} \backslash X \in \mathcal{T}_{\mathcal{I}}$. It follows that $X \in \mathcal{B} \triangle \mathbb{K}$, which contradicts the fact that $X \notin \mathcal{B} \triangle \mathbb{K}$. The proof of the case that $\mathcal{J} \supset \mathbb{L}$ runs in the same way.

The following theorem gives us another property of invariant pairs having the density property.

Theorem 2.18. If invariant pairs $\left(\mathcal{S}_{1}, \mathcal{J}\right),\left(\mathcal{S}_{2}, \mathcal{J}\right)$, having the density property generate the $\mathcal{J}$-density topologies $\mathcal{T}_{\mathcal{J}}^{1}$ and $\mathcal{T}_{\mathcal{J}}^{2}$, respectively, then

$$
\mathcal{T}_{\mathcal{J}}^{1}=\mathcal{T}_{\mathcal{J}}^{2} \Longleftrightarrow \mathcal{S}_{1}=\mathcal{S}_{2}
$$

Proof. Sufficiency is obvious.

Necessity. If $X \in \mathcal{S}_{1}$, then $\Phi_{\mathcal{J}}(X) \in \mathcal{T}_{\mathcal{J}}^{1}$ because, by the $\mathcal{J}$-density property, we have that $\Phi_{\mathcal{J}}(X) \in \mathcal{S}_{1}$ and $\Phi_{\mathcal{J}}(X) \subset \Phi_{\mathcal{J}}\left(\Phi_{\mathcal{J}}(X)\right)$. Since $\mathcal{T}_{\mathcal{J}}^{1}=\mathcal{T}_{\mathcal{J}}^{2}$, therefore $\Phi_{\mathcal{J}}(X) \in \mathcal{T}_{\mathcal{J}}^{2}$. Simultaneously, $\Phi_{\mathcal{J}}(X) \triangle X \in \mathcal{J}$. Therefore $X \in \mathcal{S}_{2}$. The proof of the case when $\mathcal{S}_{2} \subset \mathcal{S}_{1}$ runs in the same way.

Corollary 2.19. In the family of invariant $\sigma$-algebras over $\mathbb{R}$ the unique $\sigma$ algebra $\mathcal{S}$ such that the invariant pair $(\mathcal{S}, \mathbb{K})$ has the $\mathbb{K}$-density property and yields the $\mathbb{K}$-density topology identical with $\mathcal{T}_{\mathcal{I}}$ is the family of sets having the Baire property.

Corollary 2.20. In the family of invariant $\sigma$-algebras over $\mathbb{R}$ the unique $\sigma$-algebra $\mathcal{S}$ such that the invariant pair $(\mathcal{S}, \mathbb{L})$ has the $\mathbb{L}$-density property and yields the $\mathbb{L}$-density topology identical with $\mathcal{T}_{d}$ is the family of Lebesgue measurable sets. 


\section{The separation axioms of the density topologies}

We are going to present some properties of the $\mathcal{J}$-density topology $\mathcal{T}_{\mathcal{J}}$ in the aspect of separation axioms. Our results will mostly concern the $\sigma$-ideals controlled by measure and category.

Property 3.1. The space $\left(\mathbb{R}, \mathcal{T}_{\mathcal{J}}\right)$, where $\mathcal{T}_{\mathcal{J}}$ is the $\mathcal{J}$-density topology generated by the invariant pair $(\mathcal{S}, \mathcal{J})$, is Hausdorff.

Proof. By Proposition 1.10, $\mathcal{T}_{0} \subset \mathcal{T}_{\mathcal{J}}$. Hence $\left(\mathbb{R}, \mathcal{T}_{\mathcal{J}}\right)$ is Hausdorff.

Property 3.2. If a $\sigma$-ideal $\mathcal{J}$ is controlled by category, then the topological space $\left(\mathbb{R}, \mathcal{T}_{\mathcal{J}}\right)$ where $\mathcal{T}_{\mathcal{J}}$ is the $\mathcal{J}$-density topology generated by the pair $(\mathcal{S}(\mathcal{J}), \mathcal{J})$ is not regular.

Proof. Case I. Let us suppose that $\mathcal{J} \subset \mathbb{K}$. Let us observe that the set $Q$ of rational numbers is $\mathcal{T}_{\mathcal{J}}$-closed. If $\mathcal{J} \supset \mathcal{J}_{w}$, then it is clear that $\Phi_{\mathcal{J}}(\mathbb{R} \backslash Q)=$ $\mathbb{R}$. Hence $\mathbb{R} \backslash Q$ is $\mathcal{T}_{\mathcal{J}}$-open and $Q$ is $\mathcal{T}_{\mathcal{J}}$-closed. Let $\mathcal{J}=\mathcal{J}_{0}$. We show that $\Phi_{\mathcal{J}_{0}}(\mathbb{R} \backslash Q)=\mathbb{R} \backslash Q$. By Lemma 2.6, we have that $\Phi_{\mathcal{J}_{0}}(R \backslash Q) \subset \mathbb{R} \backslash Q$. Let $x \in \mathbb{R} \backslash Q$. We prove that $x \in \Phi_{\mathcal{J}_{0}}(\mathbb{R} \backslash Q)$. It suffices to show that, for an arbitrary sequence $\left\{n_{i}\right\}_{i \in \mathbb{N}}$ of positive integers, we have

$$
[-1,1] \subset n_{i}((\mathbb{R} \backslash Q)-x) .
$$

For any $i \in \mathbb{N}$ and $\alpha \in[-1,1] \cap Q$, it follows that

$$
\frac{\alpha}{n_{i}}+x \in \mathbb{R} \backslash Q
$$

Let us notice that, for each $\alpha \in[-1,1] \backslash Q$, the set

$$
A_{\alpha}=\left\{i \in \mathbb{N}: \frac{\alpha}{n_{i}}+x \notin \mathbb{R} \backslash Q\right\} .
$$

is at most a singleton. Indeed, suppose that there are $i_{1}, i_{2} \in \mathbb{N}, i_{1} \neq i_{2}$, and $\alpha / n_{i_{1}}+x=q_{1}$ and $\alpha / n_{i_{2}}+x=q_{2}, q_{1}, q_{2} \in Q$. Hence $\alpha\left(1 / n_{i_{1}}-1 / n_{i_{2}}\right)=$ $q_{1}-q_{2}$, contrary to the fact that $\alpha$ is an irrational number. Thus there exists a positive integer $k \in A_{\alpha}$ such that, for $i \geq k, \alpha / n_{i}+x \in \mathbb{R} \backslash Q$. Therefore

$$
\alpha \in n_{i}((\mathbb{R} \backslash Q)-x)
$$

and the condition $(*)$ is satisfied. We have obtained that $Q$ is closed in an arbitrary topology $\mathcal{T}_{\mathcal{J}}$.

Further, we prove that, for any $x \notin Q$, the sets $\{x\}$ and $Q$ cannot be

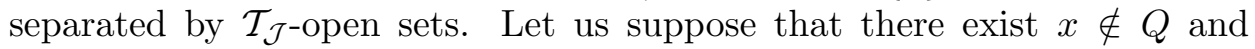
$\mathcal{T}_{\mathcal{J}}$-open sets $V_{x} \ni x$ and $V \supset Q$, such that $V_{x} \cap V=\emptyset$. It is clear that $\mathcal{S}(\mathcal{J}) \subset \mathcal{B} a$, because the pair $(\mathcal{B} a, \mathcal{J})$ is invariant and yields the $\mathcal{J}$-density 
topology. Since $\mathcal{T}_{\mathcal{J}} \subset \mathcal{S}(\mathcal{J}) \subset \mathcal{B} a$, the sets $V_{x}, V$ have the Baire property. Also,

$$
V_{x} \subset \Phi_{\mathcal{J}}\left(V_{x}\right) \subset \Phi_{\mathbb{K}}\left(V_{x}\right)
$$

and

$$
V \subset \Phi_{\mathcal{J}}(V) \subset \Phi_{\mathbb{K}}(V)
$$

Hence the nonempty sets $V_{x}$ and $V$ are open in the $\mathcal{I}$-density topology. This implies that $V_{x} \notin \mathbb{K}$ and $V \notin \mathbb{K}$. Now, we prove that each open set $V$ in the $\mathcal{I}$-density topology and containing a dense set $D$ is residual. First, we show that, for every nonempty open set $W, W \cap V \notin \mathbb{K}$. Since $W \cap D \neq \emptyset$, there exist $x \in V$ and a positive number $\delta$, such that $(x-\delta, x+\delta) \subset W$. Hence $V \cap(x-\delta, x+\delta) \notin \mathbb{K}$. Therefore $V \cap W \notin \mathbb{K}$. The set $V$ having the Baire property has the form $V=A \cup B$, where $A \in G_{\delta}$ and $B \in \mathbb{K}$. Since $V \cap W \notin \mathbb{K}$, therefore $A \cap W \neq \emptyset$. This means that $A$ is residual and thus $V$ is residual. So, $V \cap V_{x} \neq \emptyset$, contrary to the fact that $V \cap V_{x}=\emptyset$.

Case II. $\mathbb{K} \subset \mathcal{J}$. By Theorem $2.17, \mathcal{S}(\mathcal{J})=\mathcal{B} \triangle \mathcal{J}$. Similarly as in the previous case, we prove that, for any $x \notin Q$, the sets $\{x\}$ and $Q$ cannot be separated by $\mathcal{T}_{\mathcal{J}}$-open sets. Let us suppose that there exist $x \notin Q$ and $\mathcal{T}_{\mathcal{J}}$-open sets $V_{x} \ni x$ and $V \supset Q$, such that $V_{x} \cap V=\emptyset$. Since $\mathcal{T}_{\mathcal{J}} \subset \mathcal{B} \triangle \mathcal{J}$, therefore $V_{x}, V \in \mathcal{B} \triangle \mathcal{J}$. It is clear that $V_{x} \notin \mathcal{J}$. Hence $V_{x} \notin \mathbb{K}$. Also, $Q \subset V \subset \Phi_{\mathcal{J}}(V)$. Note that

$$
\mathcal{B} \triangle \mathcal{J}=\left\{X \subset \mathbb{R}: X=W \triangle Z, \quad W \in \mathcal{T}_{0}, \quad Z \in \mathcal{J}\right\} .
$$

Hence $V=W \triangle Z$, where $W \in \mathcal{T}_{0}$ and $Z \in \mathcal{J}$. Thus $\Phi_{\mathcal{J}}(V)=\Phi_{\mathcal{J}}(W)$. By Proposition 1.8 and 1.10, we have that $W \subset \Phi_{\mathcal{J}}(W) \subset \bar{W}$. Theorefore $\Phi_{\mathcal{J}}(W)=W \cup K$, where $K \in \mathbb{K}$. This implies that $Q \subset V \subset \Phi_{\mathcal{J}}(V)=$ $W \cup K$. We see that the set $\Phi_{\mathcal{J}}(V)$ has the Baire property. For every nonempty open set $U, U \cap V \notin \mathcal{J}$ since, otherwise,

$$
\emptyset \neq U \cap Q \subset U \cap V \subset \Phi_{\mathcal{J}}(U) \cap \Phi_{\mathcal{J}}(V)=\Phi_{\mathcal{J}}(U \cap V)=\emptyset .
$$

So, $U \cap W \notin \mathcal{J}$. Then $U \cap W \neq \emptyset$. Hence $W$ is dense and open. Thus $\Phi_{\mathcal{J}}(V)$ is residual. Then

$$
\emptyset \neq V_{x} \cap \Phi_{\mathcal{J}}(V) \subset \Phi_{\mathcal{J}}\left(V_{x}\right) \cap \Phi_{\mathcal{J}}(V)=\Phi_{\mathcal{J}}\left(V_{x} \cap V\right) .
$$

Hence $V_{x} \cap V \neq \emptyset$.

Property 3.3. The space $\left(\mathbb{R}, \mathcal{T}_{\mathcal{J}}\right)$, where $\mathcal{T}_{\mathcal{J}}$ is the $\mathcal{J}$-density topology generated by an invariant pair $(\mathcal{S}, \mathcal{J})$ does not possess the Lindelöf property.

Proof. According to Lemma 2.7 there exists a nonempty perfect set $F$ such that, for each $x \in F$, we have $V_{x}=(\mathbb{R} \backslash F) \cup\{x\} \in \mathcal{T}_{\mathcal{J}}$. Hence the family $\left\{V_{x}\right\}_{x \in F}$ is a covering of $\mathbb{R}$, but it has no countable subcovering of $\mathbb{R}$. 
Property 3.4. Let $\mathcal{T}_{\mathcal{J}}$ be the $\mathcal{J}$-density topology generated by an invariant pair $(\mathcal{S}, \mathcal{J})$. Then the space $\left(\mathbb{R}, \mathcal{T}_{\mathcal{J}}\right)$ is not separable.

Proof. Let $\mathcal{T}_{\mathcal{J}}$ be the $\mathcal{J}$-density topology generated by an invariant pair $(\mathcal{S}, \mathcal{J})$ and let $\mathcal{T}_{\mathcal{J}_{0}}$ be the $\mathcal{J}_{0}$-density topology generated by the invariant pair $(\mathcal{S}(\mathcal{J}), \mathcal{J})$. It is clear that $\mathcal{S}\left(\mathcal{J}_{0}\right) \subset \mathcal{S}$. Lemma 2.13 implies that $\mathcal{T}_{\mathcal{J}_{0}} \subset \mathcal{T}_{\mathcal{J}}$. Therefore it is sufficient to prove that the space $\left(\mathbb{R}, \mathcal{T}_{\mathcal{J}_{0}}\right)$ is not separable. Let $X \subset \mathbb{R}$ be a countable set. We show that there exists a nonempty set $W \in \mathcal{T}_{\mathcal{J}_{0}}$ such that $W \cap X=\emptyset$. Of course, we may assume that $X$ is infinite. Let $X=\left\{x_{1}, x_{2}, \ldots, x_{n}, \ldots\right\}$. Let us consider $\mathbb{R}$ as a vector space $\mathbb{E}$ over the field $Q$ of all rational numbers. Let $B$ be a Hamel basis of $\mathbb{E}$. For any element $x \in \mathbb{E}$ we have the unique representation $x=q_{1} b_{1}+q_{2} b_{2}+\ldots+q_{m} b_{m}$, where $m \in \mathbb{N}$ and $q_{i} \in Q \backslash\{0\}, b_{i} \in B$ for $1 \leq i \leq m$. Let $B(x)=\bigcup_{i=1}^{m}\left\{b_{i}\right\}$ and $B(X)=\bigcup_{i=1}^{\infty} B\left(x_{i}\right)$. Putting $W=\mathbb{E} \backslash \operatorname{lin}(B(X))$, where $\operatorname{lin}(B(X))$ denotes the vector space over $Q$ generated by the set $B(X)$, we have that $W \cap X=\emptyset$. We prove that $W \in \mathcal{T}_{\mathcal{J}_{0}}$. Firstly we see that $W$ is the complement of a countable set. Thus $X \in \mathcal{S}\left(\mathcal{J}_{0}\right)$ as a Borel set. Further we prove that $W \subset \Phi_{\mathcal{J}_{0}}(W)$. Let $x \in W$. Of course, $x \neq 0$. According to Lemma 1.5, we have to prove that

$$
[-1,1] \subset \bigcup_{j=1}^{\infty} \bigcap_{k=j}^{\infty} n_{k}(W-x),
$$

where $\left\{n_{k}\right\}_{k \in \mathbb{N}}$ is an increasing sequence of positive integers. Let $\alpha \in[-1,1]$. The case, where $\alpha=0$ is obvious. Suppose that $\alpha \neq 0$. Let us observe that a set $A_{\alpha}=\left\{k \in \mathbb{N}: \alpha / n_{k}+x \notin W\right\}$ is at most a singleton. Suppose to the contrary that there are $n_{k_{1}}, n_{k_{2}} \subset A_{\alpha}$ and $n_{k_{1}} \neq n_{k_{2}}$. By definition of the set $W$, we have that

and

$$
\frac{\alpha}{n_{k_{1}}}+x \in \operatorname{lin}(B(X))
$$

$$
\frac{\alpha}{n_{k_{2}}}+x \in \operatorname{lin}(B(X)) .
$$

Hence

$$
\left(n_{k_{1}}-n_{k_{2}}\right) x \in \operatorname{lin}(B(X)) .
$$

Thus $x \in \operatorname{lin}(B(X))$, contrary to the fact that $x \notin \operatorname{lin}(B(X))$. Finally, there exists a positive integer $j \in A_{\alpha}$ such that for $k \geq j, \alpha / n_{k}+x \in W$. It implies that

$$
\alpha \in \bigcup_{j=1}^{\infty} \bigcap_{k=j}^{\infty} n_{k}(W-x) .
$$


Property 3.5. Assume that $\mathcal{J}$ is an invariant $\sigma$-ideal such that $\mathbb{L} \subset \mathcal{J}$, and $\mathcal{T}_{\mathcal{J}}$ is the $\mathcal{J}$-density topology generated by an invariant pair $(\mathcal{S}(\mathcal{J}), \mathcal{J})$. The space $\left(\mathbb{R}, \mathcal{T}_{\mathcal{J}}\right)$ is regular if and only if $\mathcal{J}=\mathbb{L}$.

Proof. Sufficiency. If $\mathcal{J}=\mathbb{L}$, then $\mathcal{S}(\mathcal{J})=\mathcal{B} \Delta \mathbb{L}=\mathcal{L}$ and the $\mathcal{J}$-density topology $\mathcal{T}_{\mathcal{J}}$ is the density topology $\mathcal{T}_{d}$ which is regular (see [4]).

Necessity. Let $\mathbb{L} \subset \mathcal{J}$. Then, by Theorem 2.17, $\mathcal{S}(\mathcal{J})=\mathcal{B} \triangle \mathcal{J}$. Since $\mathbb{L} \subset \mathcal{J}$, it is clear that $\mathcal{B} \Delta \mathcal{J}=\mathcal{L} \triangle \mathcal{J}$. For any $X \in \mathcal{J}$, the inner Lebesgue measure, $l_{*}(X)=0$. Using the Marczewski method (see [11]), we can define a measure $\mu$ on the $\sigma$-algebra $\mathcal{L} \Delta \mathcal{J}$ in the following manner. Let $X \in \mathcal{L} \triangle \mathcal{J}$. Then $X=Y \triangle Z$, where $Y \in \mathcal{L}$ and $Z \in \mathcal{J}$. Putting $\mu(X)=l(Y)$, we get that $\mu$ is a correctly defined measure on $\mathcal{S}(\mathcal{J})$. Let us notice that, for the measure $\mu$ so defined, the $\sigma$-ideal $\mathcal{I}_{\mu}$ of $\mu$-null sets is of the form

$$
\mathcal{I}_{\mu}=\{X \in \mathcal{S}(\mathcal{J}): X=A \cup B, \quad A \in \mathbb{L}, \quad B \in \mathcal{J}\} .
$$

Hence $\mathcal{I}_{\mu}=\mathcal{J}$. At the same time, $\mu$ is an extension of Lebesgue measure $l$ and the pair $(\mathcal{S}(\mathcal{J}), \mathcal{J})$ is invariant. Moreover, for any $n \in \mathbb{N}, a \in \mathbb{R}$ and $X \in \mathcal{S}(\mathcal{J})$, we have $\mu(n X)=n \mu(X)$ and $\mu(X+a)=\mu(X)$. According to the above properties, we claim that a point $x \in \mathbb{R}$ is a $\mu$-density point of a set $X \in \mathcal{S}(\mathcal{J})$ if and only if it is a $\mathcal{J}$-density point of $X$. Thus

$$
\mathcal{T}_{\mathcal{J}}=\left\{X \in \mathcal{S}(\mathcal{J}): X \subset \Phi_{\mathcal{J}}(X)\right\}=\left\{X \in \mathcal{S}(\mathcal{J}): X \subset \Phi_{\mu}(X)\right\}
$$

where

$\Phi_{\mu}(X)=\{x \in \mathbb{R}: x$ is a density point of $X$ with respect to measure $\mu\}$.

By Theorem 2 from [6], we have that $\mathcal{T}_{\mathcal{J}}=\left\{X: X=A \backslash B, A \in \mathcal{T}_{d}, \mu(B)=\right.$ $0\}$. By Property 7 from [7], $\mathcal{T}_{\mathcal{J}}$ is regular if $\mathcal{T}_{\mathcal{J}}=\mathcal{T}_{d}$. We show that $\mathcal{J}=\mathbb{L}$. It is sufficient to show that $\mathcal{J} \subset \mathbb{L}$. Let $X \in \mathcal{J}$. Then $\mathbb{R} \backslash X \in \mathcal{T}_{\mathcal{J}}$. Thus $\mathbb{R} \backslash X \in \mathcal{T}_{d}$, which implies $\mathbb{R} \backslash X \in \mathcal{L}$ and $X \in \mathcal{L}$. It is clear that $0=\mu(X)=l(X)$. Hence $X \in \mathbb{L}$.

\section{References}

[1] Balcerzak, M., Hejduk, J., Density topologies for products of $\sigma$-ideals, Real Anal. Exchange 20(1) (1994-95), 163-178.

[2] Balcerzak, M., Hejduk, J., Wilczyński, W., Wroński, S., Why only measure and category?, Scient. Bull. Łódź Technical University Ser. Matematyka 695(26) (1994), 89-94.

[3] Ciesielski, K., Larson, L., Ostaszewski, K., I-density continuous functions, Mem. Amer. Math. Soc. 515 (1994).

[4] Goffman, C., Neugebauer, C., Nishiura, T., Density topology and approximate continuity, Duke Math. J. 28 (1961), 497-506. 
[5] Goffman, C., Waterman, D., Approximately continuous transformations, Proc. Amer. Math. Soc. 12 (1961), 116-121.

[6] Hejduk, J., On the density topology with resect to an extension of Lebesgue measure, Real Anal. Exchange 21(2) (1995-96), 811-816.

[7] Hejduk, J., Some properties of the density topology with respect to an extension of the Lebesgue measure, Math. Pannon. 9(2) (1998), 173-180.

[8] Hejduk, J., Kharazishvili, A. B., On density points with respect to von Neumann's topology, Real Anal. Exchange 21(1) (1995-96), 278-291.

[9] Kuczma, M., An Introduction to the Theory of Functional Equations and Inequalities, PWN, Warszawa-Katowice, 1985.

[10] Lukeš, J., Malý, J., Zajiček, L., Fine Topology Methods in Real Analysis and Potential Theory, Lecture Notes in Math. 1189, Springer Verlag, Berlin, 1986.

[11] Marczewski, E., Sur l'extension de la mesure lebesguienne, Fund. Math. 25 (1935), $551-558$.

[12] Oxtoby, J. C., Measure and Category, Springer Verlag, New York, 1980.

[13] Poreda, W., Wagner-Bojakowska, E., Wilczyński, W., A category analogue of the density topology, Fund. Math. 125 (1985), 167-173.

[14] Wagner-Bojakowska, E., Sequences of measurable functions, Fund. Math. 112 (1981), 89-102.

[15] Wilczyński, W. A category analogue of the density topology, approximate continuity and the approximate derivative, Real Anal. Exchange 10(2) (1984-85), 241-265.

[16] Wilczyński, W., A generalization of density topology, Real Anal. Exchange 8(1) (1982-83), 16-20.

\author{
JACEK HEJduk \\ FACULTY OF MATHEMATICS \\ UNIVERSITY OF ŁÓDŹ \\ BANACHA 22 \\ 90-238 ŁóDź, POLAND \\ E-MAIL:JACHEJ@MATH.UNI.LODZ.PL
}

